\title{
OPERATORS ACTING ON CERTAIN BANACH SPACES OF ANALYTIC FUNCTIONS
}

\author{
K. SEDDIGHI, K. HEDAYATIYAN and B. YOUSEFI
}

Department of Mathematics, Shiraz University

(Received January 14, 1993 and in revised form September 21, 1993)

ABSTRACT. Let $\chi$ be a reflexive Banach space of functions analytic on a plane domain $\Omega$ such that for every $\lambda$ in $\Omega$ the functional of evaluation at $\lambda$ is bounded. Assume further that $\chi$ contains the constants and $M_{z}$, multiplication by the independent variable $\mathrm{z}$, is a bounded operator on $\chi$. We give sufficient conditions for $M_{z}$ to be reflexive. In particular, we prove that the operators $M_{z}$ on $E^{p}(\Omega)$ and certain $H_{a}^{p}(\beta)$ are reflexive. We also prove that the algebra of multiplication operators on Bergman spaces is reflexive, giving a simpler proof of a result of Eschmeier.

KEY WORDS AND PHRASES. Banach spaces of analytic functions, Smirnov domain, bounded point evaluation. 1991 SUBJECT CLASSIFICATION. Primary 47B37; Secondary $47 \mathrm{~A} 25$.

\section{INTRODUCTION.}

Let $\Omega$ be a bounded domain in the complex plane C. Suppose $\chi$ is a reflexive Banach space consisting of functions that are analytic on $\Omega$ such that $1 \in \mathcal{X}$, for each $\lambda$ in $\Omega$, the functional $\mathrm{e}(\lambda): \chi \longrightarrow \mathbf{C}$ of evaluation at $\lambda$ given by $\mathrm{e}(\lambda)(f)=<f, e(\lambda)>=f(\lambda)$ is bounded, and if $\mathrm{f} \in \mathcal{X}$ then $\mathrm{zf} \in \mathcal{X}$. Note that the last condition allows us to define $M_{z}: \chi \longrightarrow \chi$ by $M_{z} f=z f, \mathrm{f} \in X$. It is easy to see that $M_{z}$ is actually a bounded operator on $X$. If $X$ is a Hilbert space, the operator $M_{z}$ and many of its properties have been studied in Shields and Wallen [1]; Bourdon and Shapiro [2]. We would like to give some sufficient conditions so that the operator $M_{z}$ becomes reflexive.

Let $\Omega$ be a bounded open set in $\mathbf{C}$ and let $p$ be a real number with $1 \leq p<\infty$. We denote by $L^{p}(\Omega)$ the $L^{p}$-space of the 2-dimensional Lebesgue measure restricted to $\Omega$. The space of analytic functions on $\Omega$ is denoted by $H(\Omega)$ and as usual $H^{\infty}(\Omega)$ is the Banach space of all bounded functions analytic on $\Omega$ equipped with the supremum norm. Each function $f \in H^{\infty}(\Omega)$ induces a bounded operator $M_{f}: L_{a}^{p}(\Omega) \longrightarrow L_{a}^{p}(\Omega), g \longrightarrow$

$f g$, where $L_{a}^{p}(\Omega)$ is the subspace of $L^{p}(\Omega)$ consisting of all analytic functions. This space is called the Bergman space.

In this article we shall prove that the algebra $B=\left\{M_{f} \mid f \in H^{\infty}(\Omega)\right\}$ is reflexive. We give a shorter proof of a result of $J$. Eschmeier [3] in case $\Omega$ is a plane domain.

\section{PRELIMINARIES.}

In this section we make a few definitions and set our notation straight. If $\mathrm{G}$ is a bounded domain in the plane, the Carathéodory hull ( $C$-hull) of $\mathrm{G}$ is the complement of the closure of the unbounded component of the complement of the closure of $\mathrm{G}$. It can be described as the interior of the set of all points $z_{0}$ in the plane such that $\left|p\left(z_{0}\right)\right| \leq$ $\sup \{|p(z)|: z \in G\}$ for all polynomials p. An open set $\mathrm{G}$ is called a Carathéodory domain if it is equal to the component of the Carathéodory hull of $\mathrm{G}$ that contains it.

For the algebra $B(X)$ of all bounded operators on a Banach space $X$, the weak operator topology (WOT) is the one in which a net $A_{\alpha}$ converges to A if $A_{\alpha} x \longrightarrow A x$ weakly, $x \in X$. 
A complex valued function $\phi$ on $\Omega$ for which $\phi \mathrm{f} \in \mathcal{X}$ for every $\mathrm{f} \in \mathcal{X}$ is called a multiplier of $\chi$ and the collection of all these multipliers is denoted by $\mathcal{M}(\chi)$ Because $M_{z}$ is a bounded operator on $\chi$, the adjoint $M_{z}^{*} \quad \chi^{*} \longrightarrow \chi^{*}$ satisfies $M_{z}{ }^{*} \mathrm{e}(\lambda) \ldots \lambda \mathrm{e}(\lambda)$. In general each multiplier $\phi$ of $\chi$ determines a multiplication operator $M_{\phi}$ defined by $M_{\phi} \mathrm{f}=\phi \mathrm{f}, \mathrm{f} \in \chi \quad$ Also $M_{\phi}{ }^{*} e(\lambda)=\phi(\lambda) e(\lambda)$ It is well known that each multiplier is a bounded analytic function, Shields and Wallen $[1]$ Indeed $|\phi(\lambda)| \leq|| M_{\phi} \|$ for each $\lambda$ in $\Omega$ Also $M_{\phi} 1=\phi \in \chi \subset H(\Omega)$ So $\phi$ is a bounded analytic function

Recall that if $\mathcal{E}$ is a subalgebra of $B(\mathcal{X})$ containing the identity operator, then $\operatorname{Lat}(\mathcal{E})$ is by definition the lattice of all invariant subspaces of $\mathcal{E}$, and $\operatorname{Alg} \operatorname{Lat}(\mathcal{E})$ is the algebra of all operators $B$ in $B(\mathcal{X})$ such that $\operatorname{Lat}(\varepsilon) \subset \operatorname{Lat}(B)$ We say that $\varepsilon$ is reflexive if $\mathcal{E}=\operatorname{Alg} \operatorname{Lat}(\mathcal{E})$ Obviously a reflexive algebra $\mathcal{E}$ is (WOT)-closed An operator $A$ in $B(X)$ is sald to be reflexive if $A \lg \operatorname{Lat}(A)=W(A)$, where $W(A)$ is the smallest subalgebra of $B(X)$ that contains $A$ and the identity $I$ and is closed in the weak operator topology.

Let $A \in A l g \operatorname{Lat}\left(M_{z}\right)$ and let $\mathcal{M}$ be a weak star closed invariant subspace of $M_{z}^{*}$ in $\chi^{*}$ Then ${ }^{\perp} \mathcal{M} \in \operatorname{Lat}\left(M_{z}\right)$ and hence ${ }^{\perp} \mathcal{M} \in \operatorname{Lat}(A)$. Therefore, $\left({ }^{\perp} \mathcal{M}\right)^{\perp} \in \operatorname{Lat}\left(A^{*}\right)$. Since $\mathcal{M}$ is weak star closed, $\mathcal{M} \in \operatorname{Lat}\left(A^{*}\right)$. Now the one-dimensional span of $e(\lambda)$ is invariant under $M_{z}^{*}$. Therefore, it is invariant under $A^{*}$. We write $A^{*} e(\lambda)=\phi(\lambda) e(\lambda), \lambda \in \Omega$. So $<f, A^{*} e(\lambda)>=\phi(\lambda) f(\lambda) ; \lambda \in \Omega$. Using the Hahn-Banach theorem we see that the linear span of $\{e(\lambda)\}_{\lambda \in \Omega}$ is weak star dense in $\chi^{*}$. Thus $\phi \in \mathcal{M}(X)$ and $\mathbf{A}=M_{\phi}$.

\section{REFLEXIVITY.}

In this section we consider a Banach space of functions analytic on a Carathéodory domain and give sufficient conditions for the operator of multiplication to be reflexive. A circular domain is also considered.

THEOREM 1. Let $\Omega$ be a Carathéodory domain each point of which is a bounded point evaluation for a reflexive Banach space $\chi$ of functions analytic on $\Omega$ which contains the constant functions and admits $M_{z}$ as a bounded operator. Furthermore, if $\left\|M_{p}\right\| \leq C\|p\|_{\Omega}$ for every polynomial $\mathrm{p}$, then $M_{z}$ is reflexive.

PROOF. Let A $\in$ Alg $\operatorname{Lat}\left(M_{z}\right)$. Then $\mathrm{A}=M_{\phi}$ for some multiplier $\phi \in H^{\infty}(\Omega)$. Let $\left\{p_{n}\right\}$ be a sequence of polynomials such that sup $\left\|p_{n}\right\| \Omega \leq M$ for some constant $\mathrm{M}$ and $p_{n}(z) \longrightarrow \phi(z), z \in \Omega$. Then $\left\|M_{p_{n}}\right\| \leq C\left\|p_{n}\right\|_{\Omega} \leq C M$. Since $\chi$ is reflexive, the unit ball of $X$ is weakly compact. Therefore, the unit ball of $B(X)$ is (WOT) compact. We may assume, by passing to a subsequence if necessary, that $M_{p_{n}} \longrightarrow X$ (WOT) for some operator X. Thus $M_{p_{n}}^{*} e(\lambda) \longrightarrow X^{*} e(\lambda)$ in the weak star topology. On the other hand $M_{p_{n}}^{*} e(\lambda)=p_{n}(\lambda) e(\lambda) \longrightarrow \phi(\lambda) e(\lambda)=M_{\phi}^{*} e(\lambda)$ in the weak star topology for every $\lambda \in \Omega$. Therefore, $X^{*} e_{\lambda}=M_{\phi}^{*} e_{\lambda}$ and thus $X^{*}=M_{\phi}^{*}$. Hence $\mathrm{X}=M_{\phi}$ on $\chi$, which implies that $A \in W\left(M_{z}\right)$ and $M_{z}$ is reflexive.

Now we use the technique of the proof of Theorem 1 to give a short proof of a result of Eschmeier [3]. We let B $=\left\{M_{f} \mid f \in H^{\infty}(\Omega)\right\}$, where $\Omega$ is a bounded domain and $M_{f}$ acts on $L_{a}^{p}(\Omega)$.

THEOREM 2. The algebra $B$ is reflexive.

PROOF. Clearly B $\subseteq$ Alg Lat(B). Let $A \in$ Alg Lat(B). Because the one dimensional span of $\mathrm{e}(\lambda)$ is invariant under $M_{f}^{*}$ for all $\mathrm{f}$ in $H^{\infty}(\Omega)$, it is invariant under $A^{*}$, and therefore $\mathrm{A}=M_{\phi}$ for some multiplier $\phi$. Thus $\mathrm{B}$ is a reflexive algebra. 1 .

Next we give a few examples of Banach spaces satisfying the hypothesis of Theorem

EXAMPLE 3. Let $\Omega$ be an arbitrary simply connected Smirnov domain. Let $1<p<\infty$. Define $E^{p}(\Omega)$ to be the set of all analytic functions $f$ on $\Omega$ such that there exists a sequence of rectifiable Jordan curves $C_{1}, C_{2}, \cdots$ in $\Omega$, tending to the boundary in the sense that $C_{n}$ eventually surrounds each compact subdomain of $\Omega$, such that $\int_{C_{n}}|f(z)|^{p}|d z| \leq M<\infty$. For a good source on $E^{p}(\Omega)$ see Duren [4, Chapter 10]. Every function $\mathrm{f}$ of class $E^{p}(\Omega)$ has a nontangential limit almost everywhere on $\partial \Omega$, which does not vanish on a set of positive measure unless $\mathrm{f}(\mathrm{z}) \equiv 0$. Furthermore, $\int_{\partial \Omega}|f(z)|^{p}|d z|<$ $\infty$. It is convenient to identify $E^{p}(\Omega)$ with its set of boundary functions. Thus $E^{p}(\Omega)$ is a closed subspace of $L^{p}(\partial \Omega)$ which contains the set of all polynomials, and hence its closure. Hence $E^{p}(\Omega)$ is a reflexive Banach space.

Clearly $M_{z}$ is bounded and $\left\|M_{p}\right\| \leq\|p\|_{\Omega}$ for all polynomials p. Now we show that 
each point of $\Omega$ is a bounded point evaluation for $E^{p}(\Omega)$ For a fixed $z$ in $\Omega$, choose $C>0$ such that $\operatorname{dist}(\mathrm{z}, \partial \Omega) \geq \mathrm{C}$ Let $\mathrm{f} \in E^{p}(\Omega)$ Then $f^{p} \in E^{1}(\Omega)$ and it has a Cauchy representation

$$
f^{p}(z)=\frac{1}{2 \pi \imath} \int_{3 \Omega} \frac{f^{p}(\varsigma)}{\zeta-z} d \zeta, \quad z \in \Omega
$$

Therefore $|f(z)|^{p} \leq(1 / 2 \pi C)\|f\|^{p}$ Thus each point of $\Omega$ is a bounded point evaluation for $E^{p}(\Omega)$. Finally, by Theorem $31, M_{z}$ is reflexive

Further examples of Banach spaces satisfying the hypothesis of Theorem 1 will be presented We also deduce that $M_{z}$ acting on these spaces are reflexive. We begin with a definition.

DEFINITION 4. Let $1<p<\infty$ and let $\{\beta(n)\}$ be a sequence of positive numbers with $\beta(0)=1$. We consider the space of sequences $f=\{\hat{f}(n)\}$ such that

$$
\|f\|_{p}^{p}=\sum_{n=0}^{\infty}|\hat{f}(n)|^{p}[\beta(n)]^{p}<\infty .
$$

We shall use the formal notation $f(z)=\sum_{n=o}^{\infty} \hat{f}(n) z^{n}$ for $\mathrm{z} \in \mathbf{D}$ the unit disc in $\mathbf{C}$ (See Shields [5] for $\mathrm{p}=2$.). Let $H^{p}(\beta)=\left\{f \mid f(z)=\sum_{n=0}^{\infty} \hat{f}(n) z^{n} ;\|f\|_{p}<\infty\right\}$ and $H_{a}^{p}(\beta)=\left\{f \in H^{p}(\beta) \mid f(z)=\sum_{n=0}^{\infty} \hat{f}(n) z^{n}\right.$ is convergent in $\left.\mathbf{D}\right\}$.

REMARK 5. Define the $\sigma$-finite measure $\mu$ on the positive integers by $\mu(K)=$ $\sum_{n \in K} \beta(n)^{p}, K \subseteq \mathbf{N}$. Because $H^{p}(\beta) \cong L^{p}(\mu)$ we conclude that $H^{p}(\beta)$ is indeed a reflexive Banach space.

REMARK 6. If $\{\beta(n+1) / \beta(n)\}$ is bounded, the operator of multiplication by $z$ is a bounded operator on $H^{p}(\beta)$. Indeed $\left\|M_{z}\right\|=\sup _{n} \frac{\beta(n+1)}{\beta(n)}$.

In the following examples let $q$ be the conjugate of $p(1 / p+1 / q=1)$.

EXAMPLE 7. Let $\{1 / \beta(n)\} \in \ell^{q}$. If $\mathrm{f} \in H^{p}(\beta)$ and $\lambda \in \mathbf{D}$, we have

$$
|f(\lambda)|=\left|\sum_{n=0}^{\infty} \hat{f}(n) \lambda^{n}\right| \leq\left(\sum_{n=0}^{\infty}|\hat{f}(n)|^{p}[\beta(n)]^{p}\right)^{1 / p}\left(\sum_{n=0}^{\infty} \frac{|\lambda|^{n q}}{\beta(n)^{q}}\right)^{1 / q} .
$$

Therefore, $\mathrm{f}$ is analytic and $\|f\|_{\mathrm{D}} \leq\left\|\left\{\frac{1}{\beta(n)}\right\}\right\|_{q}\|f\|_{p}$. We conclude that $H_{a}^{p}(\beta)=$ $H^{p}(\beta) \subset H^{\infty}$. Furthermore, each point of $\mathbf{D}$ is a bounded point evaluation for $H^{p}(\beta)$ and also convergence in $H^{p}(\beta)$ implies uniform convergence on $\mathbf{D}$.

EXAMPLE 8. In Example 7 assume $\beta(n) \geq 1$ for all $n$. In this case, it follows from (1) that $\|f\|_{K} \leq C\|f\|_{p}$ for any compact $K \subset \mathbf{D}$, where $C$ depends on $K$.

EXAMPLE 9. Let $p>1$. Also suppose that $\sup _{n} \frac{\beta(n+1)}{\beta(n)}=1$ (e.g. $\beta(n)=1$ or $\beta$ (n) $=1+1 / \mathrm{n})$. It can easily be seen that $\overline{\mathbf{D}}=\sigma\left(M_{z}\right)$. Since $M_{z}$ is a contraction, $\overline{\mathbf{D}}$ is a spectral set for $M_{z}$ and $\left\|M_{p}\right\| \leq\|p\|_{D}$ for every polynomial p. By Theorem $1, M_{z}$ acting on $H_{a}^{p}(\beta)$ is reflexive.

The domains considered in Theorem 1 were Carathéodory domains. We now extend the conclusion of this Theorem to a circular domain, that is, any domain obtained by removing a finite number of disjoint subdiscs from the open unit disc. In Seddighi and Yousefi [6] we have proved the analogue of the following theorem for a Hilbert subspace of $H(\Omega)$. For the proof combine the techniques of the proof of Theorem 1 with Seddighi and Yousefi [6, Theorem 5.1].

THEOREM 10. Let $\Omega$ be a circular domain each point of which is a bounded point evaluation for a reflexive Banach subspace $X$ of $\mathrm{H}(\Omega)$ which contains the constants and admits multiplication by the independent variable $\mathrm{z}, M_{\mathbf{z}}$, as a bounded operator. Furthermore, suppose that $\left\|M_{p}\right\| \leq\|p\|_{\Omega}$ for every polynomial p. Then $M_{z}$ is reflexive.

We present an example of a Banach space satisfying the hypothesis of Theorem 10.

EXAMPLE 11. Let $\Omega$ be a circular domain and $1<p<\infty$. Since $L_{a}^{p}(\Omega)$ is closed in $L^{p}(\Omega), L_{a}^{p}(\Omega)$ is reflexive. By Lemma 3.7 of Garnett [7] every point of $\Omega$ is a bounded point evaluation for $L_{a}^{p}(\Omega)$. It is also clear that $\left\|M_{p}\right\| \leq\|p\|_{\Omega}$ for every polynomial p. By Theorem 4 the multiplication operator $M_{z}$ on $L_{a}^{p}(\Omega)$ is reflexive.

ACKNOWLEDGEMENT. Research of the first author was partially supported by a grant (no. 67-SC-520-276) from Shiraz University Research Council. 


\section{REFERENCES}

1. SHIELDS, A.L. and WALLEN, L. The Commutants of certain Hilbert space operators, Indiana Univ. Math. J. 20(1971), 777-788.

2. BOURDON, P.S. and SHAPIRO, J.H. Spectral synthesis and common cyclic vectors, Michigan Math. J. 37 (1990),71-90.

3. ESCHMEIER, J. Multiplication operators on Bergman spaces are reflexive, Operator Theory : Advances and Applications 43 (1990), 165-184.

4. DUREN, P. $H^{p}$ Spaces, Academic Press, New York 1972.

5. SHIELDS, A. Weighted shift operators and analytic function theory, Topics in operator theory, Math. Surveys, No.13, Amer. Math. Soc., Providence, R. I., 49-128

6. SEDDIGHI, K. and YOUSEFI, B. On the reflexivity of operators on function spaces, Proc. Amer. Math. Soc. 116(1992), 45-52.

7. GARNETT, J.B. Bounded Analytic Functions, Academic Press, New York 1981. 


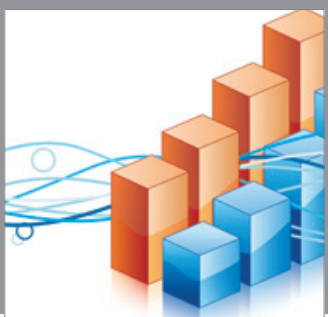

Advances in

Operations Research

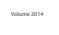

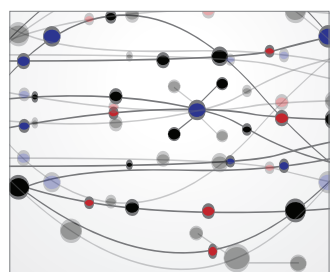

\section{The Scientific} World Journal
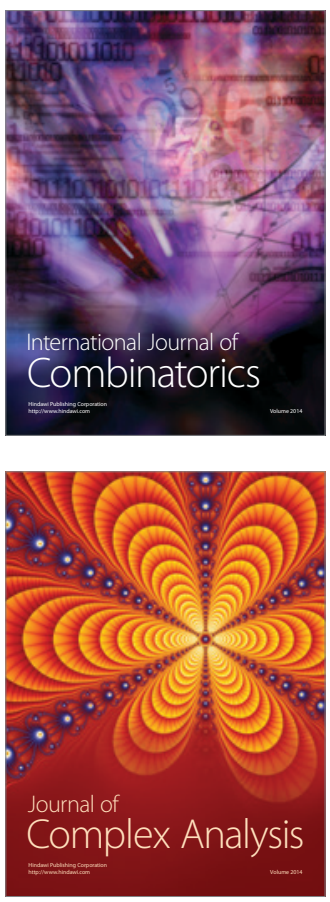

International Journal of

Mathematics and

Mathematical

Sciences
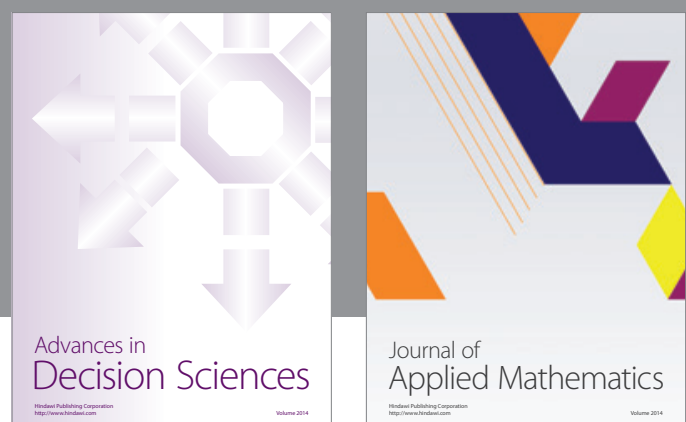

Journal of

Applied Mathematics
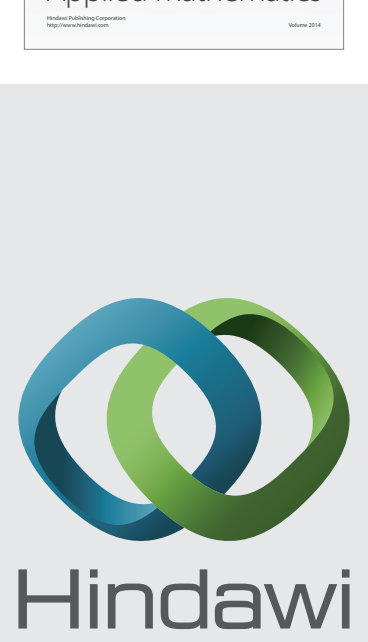

Submit your manuscripts at http://www.hindawi.com
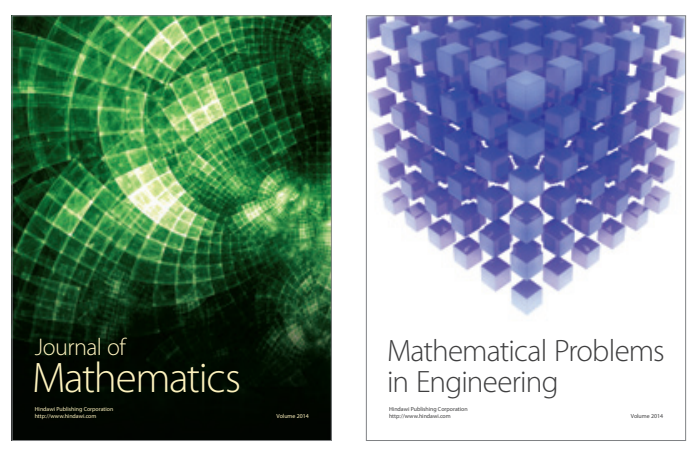

Mathematical Problems in Engineering
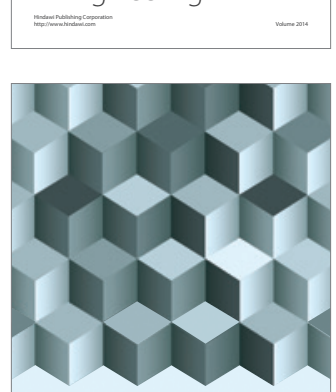

Journal of

Function Spaces
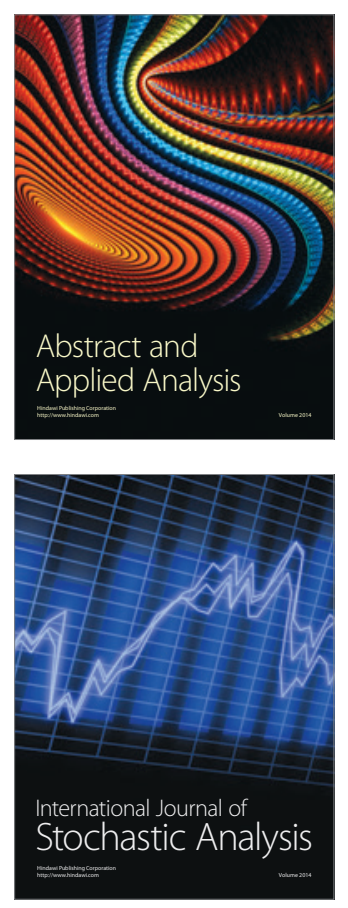

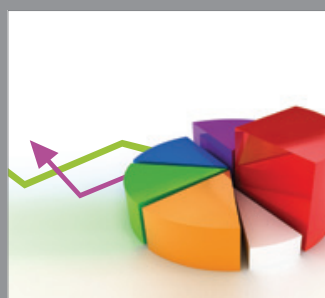

ournal of

Probability and Statistics

Promensencen
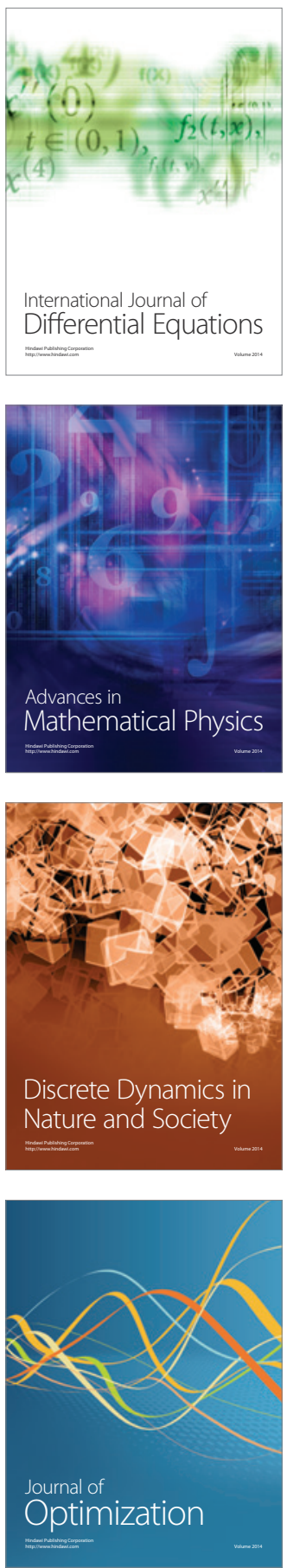